\title{
A VARIABLE EXPONENT DIFFUSION PROBLEM OF CONCAVE-CONVEX NATURE
}

\author{
JORGE GARCÍA-MELIÁN, JULIO D. ROSSI AND JOSÉ C. SABINA DE LIS
}

ABSTRACT. This paper deals with the problem

$$
\left\{\begin{array}{cl}
-\Delta u=\lambda u^{q(x)} & x \in \Omega \\
u=0 & x \in \partial \Omega,
\end{array}\right.
$$

where $\Omega \subset \mathbb{R}^{N}$ is a bounded smooth domain, $\lambda>0$ is a parameter and the exponent $q(x)$ is a continuous positive function that takes values both greater than and less than one in $\bar{\Omega}$. It is therefore a kind of concave-convex problem where the presence of the interphase $q=1$ in $\bar{\Omega}$ poses some new difficulties to be tackled. The results proved is this work are the existence of $\lambda^{*}>0$ such that no positive solutions are possible for $\lambda>\lambda^{*}$, the existence and structural properties of a branch of minimal solutions, $u_{\lambda}, 0<\lambda<\lambda^{*}$, and, finally, the existence for all $\lambda \in\left(0, \lambda^{*}\right)$ of a second positive solution.

\section{INTRODUCTION}

This work is devoted to the analysis of positive solutions to the semilinear boundary value problem:

$$
\begin{cases}-\Delta u=\lambda u^{q(x)} & x \in \Omega \\ u=0 & x \in \partial \Omega,\end{cases}
$$

where $\Omega \subset \mathbb{R}^{N}$ is a bounded smooth domain and $\lambda>0$ is a bifurcation parameter. The exponent $q(x)$ is assumed to be a positive function $q \in$ $C^{\gamma}(\bar{\Omega})$, the main feature being the fact that

$$
0<\inf _{\Omega} q<1<\sup _{\Omega} q .
$$

Thus, problem (1.1) can be regarded in its own right as a sort of "concaveconvex" problem. To simplify the exposition, it will be also assumed that $N \geq 3$ and that (1.1) is subcritical, i. e.,

$$
q(x) \leq \frac{N+2}{N-2} \quad x \in \Omega .
$$

Reaction-Diffusion systems constitutes an important and active area in the theory of nonlinear problems (see [14, [40, 34] and [33] for a global overview). One of its multiple branches is constituted by concave-convex problems, which have received some interest in the literature in recent times, including several kinds of boundary conditions and generalizations to other

Date: October 11, 2014.

Key words and phrases. Concave-convex nonlinearities, sub and supersolutions, a priori estimates, degree theory. 
operators such as the $p$-Laplacian or fully nonlinear uniformly elliptic operators. The subject goes back to the pioneering works [29], [5], 16] and [17. However, 3] is regarded as a first detailed analysis of the main properties of such type of problems, especially its bifurcation diagrams (see also [29], Section 1.1). Later extensions are [4, [15, that deal with Dirichlet conditions and the $p$-Laplacian operator; [10, dedicated to fully nonlinear uniformly elliptic operators with Dirichlet boundary conditions; [18], [37, dealing with flux-type nonlinear boundary conditions and source nonlinearities; 21] handling concave-convex terms of absorption nature and [11, where a combination of concave absorption with a convex source leads to an interesting free boundary problem in $\mathbb{R}^{N}$. Of course, all these works are only a reduced sample of the previous research on the topic.

On the other hand, we would also like to mention the previous papers [13], [19], 20], 27], 30], 31] and [35], which have dealt with different types of elliptic problems involving variable exponents. However, at the best of our knowledge, no concave-convex problems such as (1.1) have been considered before in the literature.

A main feature of our problem (1.1) is the presence of the interphase $q=1$ in $\bar{\Omega}$. This fact raises subtle technical problems in several key points of the analysis. For example, let us mention the validity of the Palais-Smale condition, the existence of extremal solutions and the uniqueness of the small amplitude solutions for $\lambda$ close to zero among others. Such issues are simpler to handle when the concave nonlinearity is well-separated from the convex one which is just the case in 3 .

To progress further, a more precise hypothesis on the exponent $q$ should be introduced. It is implicit in $(1.2)$ that the open sets $\Omega_{-}:=\{x \in \Omega$ : $q(x)<1\}, \Omega_{+}=\{x \in \Omega: q(x)>1\}$ are nonempty. It will be assumed that $\Omega_{ \pm}$are the union of finitely many smooth domains. In addition, and to avoid unnecessary extra technicalities, we will also suppose that both $\Gamma_{ \pm}:=$ $\partial \Omega_{ \pm} \cap \Omega$ consist of closed manifolds, i.e., $\partial \Omega_{ \pm} \cap \partial \Omega$ are whole components of $\partial \Omega$. Therefore, $\partial \Omega_{ \pm} \cap \partial \Omega$ and $\Gamma_{ \pm}$are disjoint. All such conditions on $q$ -including (1.3) - will be henceforth referred to as $(H)_{q}$.

We state now our main result.

Theorem 1. Let $q$ be a positive function in $C^{\gamma}(\bar{\Omega})$ satisfying $(H)_{q}$. Then, problem 1.1

$$
\begin{cases}-\Delta u=\lambda u^{q(x)} & x \in \Omega \\ u=0 & x \in \partial \Omega,\end{cases}
$$

with $\lambda>0$, has the following properties:

i) There exists $\lambda^{*}>0$ such that no positive weak solutions exist if $\lambda>\lambda^{*}$.

ii) For every

$$
0<\lambda<\lambda^{*}
$$

there exists a minimal (classical) solution $u_{\lambda} \in C^{2, \alpha}(\bar{\Omega})$ for some $0<\alpha<1$. In addition,

$$
u_{\lambda} \rightarrow 0 \quad \text { as } \lambda \rightarrow 0+
$$


in $C^{2}(\bar{\Omega})$, meanwhile the mapping $\lambda \mapsto u_{\lambda}$ from $\left(0, \lambda^{*}\right)$ with values in $C^{2}(\bar{\Omega})$ is continuous from the left at every $\lambda$. Moreover, if $v_{\lambda}$ is any family of positive solutions to (1.1) such that $\left\|v_{\lambda}\right\|_{L^{\infty}(\Omega)}=O(1)$ as $\lambda \rightarrow 0$ then there exists a positive function $\varphi \in C^{1}(\Omega)$ which vanishes on $\partial \Omega$ such that

$$
v_{\lambda}(x) \leq \lambda^{\frac{1}{1-q_{-}}} \varphi(x) \quad x \in \Omega,
$$

for small $\lambda$, where $q_{-}=\inf _{\Omega} q<1$.

iii) The solution $u_{\lambda}$ is linearly stable in the sense that,

$$
\int_{\Omega}|\nabla \varphi|^{2} \geq \int_{\Omega} q(x) u_{\lambda}^{q(x)-1} \varphi^{2}
$$

for all $\varphi \in H_{0}^{1}(\Omega)$.

iv) Suppose that

$$
q(x)<\frac{N}{N-2} \quad x \in \bar{\Omega},
$$

and that either $\Omega_{+} \subset \bar{\Omega}_{+} \subset \Omega$ or $q>1$ on $\partial \Omega_{+} \cap \partial \Omega$ provided such intersection is nonempty. Then, for every $0<\lambda<\lambda^{*}$ there exists a second positive solution $v_{\lambda} \in C^{2, \alpha}(\bar{\Omega})$ for some $0<\alpha<1$. Moreover, a minimal solution $u_{\lambda^{*}}$ exists at $\lambda=\lambda^{*}$ while the limit

$$
u_{\lambda^{*}}=\lim _{\lambda \rightarrow \lambda^{*}-} u_{\lambda}
$$

holds in $C^{2}(\bar{\Omega})$.

Remarks 1.

(a) Observe that the existence of an extremal solution corresponding to $\lambda=\lambda^{*}$ is not provided in ii). Such existence is furnished in iv) under more restrictive conditions.

(b) Inequality (1.5) means that the first eigenvalue $\sigma_{1}$ of the linearization of (1.1) around $u=u_{\lambda}$ (see Lemma 5 and (2.7) below) is nonnegative.

(c) Regarding the existence and remaining properties of the minimal solution $u_{\lambda}$ it should be remarked that no restriction as 1.3 on the size of $q(x)$ is required for them to hold (see Section 1 in [3]).

A more precise description on the global structure of the branch of minimal solutions $u_{\lambda}$ is given in the next statement.

Theorem 2. There exists at most a decreasing sequence of values $\lambda_{n}$ in the interval $\left(0, \lambda^{*}\right)$ such that the following properties hold.

i) The mapping $\lambda \mapsto u_{\lambda}$ is discontinuous at every $\lambda_{n}$.

ii) For every $n$, the mapping

$$
\begin{array}{cl}
\left(\lambda_{n+1}, \lambda_{n}\right) & \longrightarrow C_{0}^{1}(\bar{\Omega}) \\
\lambda & \longmapsto u_{\lambda}
\end{array}
$$

is smooth, increasing and satisfies

$$
\sigma_{1}\left(u_{\lambda}\right)>0,
$$

for every $\lambda \in\left(\lambda_{n+1}, \lambda_{n}\right)$. This fact holds also true for the initial interval $\left(\lambda_{1}, \lambda^{*}\right)$. 
Remarks 2.

(a) A possible quite likely option, which is not discarded in Theorem 2, is the non existence of such values $\lambda_{n}$. In that case, ii) is satisfied in the whole interval $\left(0, \lambda^{*}\right)$.

(b) Relation (1.7) asserts that $u_{\lambda}$ is asymptotically stable with the possible exception of the values $\lambda=\lambda_{n}$.

The rest of the paper is organized as follows: Section 2 studies a variant (see (2.2) below) of our main problem (1.1), which is instrumental to get the existence of a minimal solution to (1.1). The smoothness of weak solutions to problem (1.1) and the eigenvalue problem associated to its linearization around a positive solution $u$ are also analyzed in Section 2. The $L^{\infty}$ estimates required to attain the existence of a second positive solution is carried out in Section 3 , Section 4 is devoted to the proof of Theorem 1, while the analysis of the structure of the branch $u_{\lambda}$ of minimal solutions is addressed in Section 5 ,

\section{Auxiliary material}

In this section, we will obtain some preliminary results, which will be instrumental in the proofs in Sections 3,4 and 5. We begin by analyzing the regularity of solutions to a slight generalization of problem (1.1).

Lemma 3. Assume that $q$ is a measurable function in $\Omega$ fulfilling the growth condition 1.3. Then, every weak solution $u \in H_{0}^{1}(\Omega)$ to the problem

$$
\begin{cases}-\Delta u=\lambda|u|^{q(x)-1} u & x \in \Omega \\ u=0 & x \in \partial \Omega,\end{cases}
$$

verifies $u \in C^{1, \beta}(\bar{\Omega})$ for every $0<\beta<1$. If in addition $q \in C^{\gamma}(\bar{\Omega})$ then $u \in C^{2, \alpha}(\bar{\Omega})$ for some $0<\alpha<1$.

Proof. The function $f(x, u)=|u|^{q(x)-1} u$ verifies a Carathéodory condition and

$$
|f(x, u)| \leq b(x)(1+|u|),
$$

for some $b \in L^{\frac{N}{2}}(\Omega)$. It follows from [8] that $u \in L^{p}(\Omega)$ for all $p>1$ and thus the same happens to $f(\cdot, u(\cdot))$. Then, it is a consequence of the $L^{p}$ theory for elliptic equations and the embedding of Sobolev spaces that $u \in C^{1, \beta}(\bar{\Omega})$ for every $0<\beta<1$ (see [24]).

On the other hand, it can checked that the Nemytskii operator $N_{f}$, $u(\cdot) \mapsto N_{f}(u)(\cdot)$, given by $N_{f}(u)(\cdot)=f(\cdot, u(\cdot))$, maps $C^{1}(\bar{\Omega})$ into $C^{\theta}(\bar{\Omega})$ with $\theta=\min \left\{\gamma, \inf _{\Omega} q\right\}$. Since $\partial \Omega$ is smooth, Schauder theory implies that $u \in C^{2, \alpha}(\bar{\Omega})$ for some $0<\alpha<1$ (we refer again to [24]).

Next we deal with an auxiliary sublinear problem related to (1.1) and obtain some of its more relevant properties.

Theorem 4. Assume that $q \in C^{\gamma}(\bar{\Omega})$ satisfies $(H)_{q}$ and consider the problem,

$$
\begin{cases}-\Delta u=\lambda \chi_{\Omega_{-}}(x) u^{q(x)} & x \in \Omega \\ u=0 & x \in \partial \Omega,\end{cases}
$$


where $\chi_{\Omega_{-}}$stands for the characteristic function of $\Omega_{-}$. Then the following assertions hold:

i) For every $\lambda>0$ problem (2.2) admits a unique weak positive solution $\tilde{u}_{\lambda} \in C^{1, \beta}(\bar{\Omega}), 0<\beta<1$ arbitrary. Moreover, the mapping $\lambda \mapsto \tilde{u}_{\lambda}$ is increasing.

ii) There exist $\lambda_{0}>0$ and a function $\varphi \in C^{1}(\bar{\Omega})$, verifying $\varphi(x)>0$ for $x \in \Omega$ and $\varphi(x)=0$ for $x \in \partial \Omega$, such that

$$
\tilde{u}_{\lambda}(x) \leq \lambda^{\frac{1}{1-q_{-}}} \varphi(x) \quad x \in \Omega,
$$

for all $0<\lambda \leq \lambda_{0}$ where $q_{-}=\inf _{\Omega} q<1$. In particular, $\tilde{u}_{\lambda} \rightarrow 0$ as $\lambda \rightarrow 0+$ in $C^{1, \beta}(\bar{\Omega})$.

iii) For all $q_{1} \in\left(\inf _{\Omega} q, 1\right)$ there exist $\lambda_{1}>0$, and a function $\psi \in C(\bar{\Omega})$, verifying $\psi(x)>0$ for $x \in \Omega, \psi(x)=0$ for $x \in \partial \Omega$, such that

$$
\tilde{u}_{\lambda}(x) \geq \lambda^{\frac{1}{1-q_{1}}} \psi(x) \quad x \in \Omega,
$$

for $\lambda \geq \lambda_{1}$. In particular, $\tilde{u}_{\lambda} \rightarrow \infty$ uniformly in compact sets of $\Omega$ as $\lambda \rightarrow \infty$.

Proof. The functional

$$
J_{0}(u)=\int_{\Omega}\left(\frac{1}{2}|\nabla u|^{2}-\frac{\lambda}{q+1} \chi_{\Omega_{-}}|u|^{q+1}\right)
$$

is well defined in $H_{0}^{1}(\Omega)$ since $\chi_{\Omega_{-}}|u|^{q+1} \leq u^{2}+1$, and it is coercive. In fact, if this is not the case there exists a sequence $\left\{u_{n}\right\}$ with $\left\|u_{n}\right\|_{H_{0}^{1}(\Omega)} \rightarrow+\infty$ and $J_{0}\left(u_{n}\right) \leq C$. Then

$$
\int_{\Omega} \frac{\lambda}{q+1} \chi_{\Omega_{-}}\left|u_{n}\right|^{q+1} \rightarrow \infty
$$

This implies that $\left\|u_{n}\right\|_{L^{2}(\Omega)} \rightarrow \infty$. By setting $v_{n}=t_{n}^{-1} u_{n}, t_{n}=\left\|u_{n}\right\|_{L^{2}(\Omega)}$ it can be checked that

$$
\int_{\Omega} \frac{\lambda}{q+1} t_{n}^{q-1} \chi_{\Omega_{-}}\left|v_{n}\right|^{q+1} \rightarrow 0
$$

Since $J_{0}\left(v_{n}\right) \leq o(1)$ it follows that $v_{n} \rightarrow 0$ in $H_{0}^{1}(\Omega)$ which is not possible since we have $\left\|v_{n}\right\|_{L^{2}(\Omega)}=1$.

On the other hand, if we denote by $\lambda_{1}$ the first Dirichlet eigenvalue of the Laplacian in $\Omega$ and by $\phi_{1}$ its corresponding associated positive eigenfunction, we have

$$
\frac{J_{0}\left(t \phi_{1}\right)}{t^{2}}=\int_{\Omega}\left(\frac{\lambda_{1}}{2} \phi^{2}-t^{q-1} \frac{\lambda}{q+1} \phi_{1}^{q+1}\right),
$$

for $t>0$. Hence the integral becomes negative if $t$ is chosen small enough. Therefore, $J_{0}$ achieves the absolute minimum at some $u \in H_{0}^{1}(\Omega), u \neq 0$, that can be assumed to be nonnegative. This function $u$ is in particular a nonnegative weak solution to 2.2 .

Observe next that an argument entirely similar to the one used in the proof of Lemma 3 shows that weak nonnegative solutions to 2.2 lie also 
in $C^{1, \beta}(\bar{\Omega})$ for every $0<\beta<1$. In particular they are positive in $\Omega$ with negative normal derivative

$$
\frac{\partial u}{\partial \nu}<0
$$

on $\partial \Omega([9])$.

To prove uniqueness, assume now that $u, v$ are weak positive solutions to (2.2). Then the following inequality holds true ([9, p. 57):

$$
I:=\left\langle\left(-\frac{\Delta u}{u}\right)-\left(-\frac{\Delta v}{v}\right), u^{2}-v^{2}\right\rangle \geq 0,
$$

and equality is achieved only when $v=c u$ for some positive constant $c$. But

$$
I=\lambda \int_{\Omega_{-}}\left(u^{q-1}-v^{q-1}\right)\left(u^{2}-v^{2}\right) \leq 0 .
$$

Thus, we get $v=c u$, and it follows that $c=1$, which shows uniqueness.

To show the monotonicity of $\tilde{u}_{\lambda}$ with respect to $\lambda$ first observe that 2.2 possesses subsolutions whose amplitude can be as small as desired. In fact, for an arbitrary ball $B \subset \bar{B} \subset \Omega_{-}$define $\underline{u}(x)=\varepsilon \phi_{1, B}(x)$ if $x \in \bar{B}, \underline{u}(x)=0$ otherwise, where $\phi_{1, B}$ is a positive normalized first Dirichlet eigenfunction in $B$ and $\varepsilon>0$ a small parameter. On the other hand $\tilde{u}_{\lambda^{\prime}}$ is a strict supersolution to (2.2) if $\lambda^{\prime}>\lambda$. Since $\underline{u}$ can be found so that $\underline{u} \leq \tilde{u}_{\lambda^{\prime}}$ we finally get $\tilde{u}_{\lambda}<\tilde{u}_{\lambda^{\prime}}$ whenever $0<\lambda<\lambda^{\prime}$. Thus, $\tilde{u}_{\lambda}$ increases with $\lambda$. This concludes the proof of part i).

To prove ii), notice that $\left\{\tilde{u}_{\lambda}\right\}$ is uniformly bounded in $L^{\infty}(\Omega)$ for $0<\lambda<$ $\lambda_{0}$. Thus, by using $L^{p}$ estimates we can conclude that $\tilde{u}_{\lambda} \rightarrow 0$ in $C^{1, \beta}(\bar{\Omega})$ (remark that the only nonnegative solution to $(2.2)$ with $\lambda=0$ is $u=0$ ). On the other hand, by taking $\lambda_{0}$ small enough to have $\tilde{u}_{\lambda} \leq 1$ for $0<\lambda \leq \lambda_{0}$ we observe that $u=\tilde{u}_{\lambda}$ satisfies,

$$
-\Delta u \leq \lambda u^{q_{-}} \quad x \in \Omega,
$$

with $q_{-}=\inf _{\Omega} q<1$. Thus $\tilde{u}_{\lambda} \leq \lambda^{\frac{1}{1-q_{-}}} \varphi$ where $\varphi$ is the solution to:

$$
\left\{\begin{array}{cl}
-\Delta \varphi=\varphi^{q_{-}} & x \in \Omega \\
\varphi=0 & x \in \partial \Omega .
\end{array}\right.
$$

This proves ii).

As for iii) we chose a smooth subdomain $D \subset \bar{D} \subset \Omega_{-}$and set $u=\hat{u}_{\lambda}$ the solution to the problem:

$$
\left\{\begin{array}{cl}
-\Delta u=\lambda u^{q(x)} & x \in D \\
u=0 & x \in \partial D .
\end{array}\right.
$$

The previous existence and uniqueness proof applies, of course, to 2.5). So, it is clear that $\tilde{u}_{\lambda} \geq \hat{u}_{\lambda}$. On the other hand, the function

$$
\underline{u}=\lambda^{\frac{1}{1-\theta}} \hat{u}_{1}
$$

defines a subsolution to 2.5) if $\theta=\inf _{D} q<1$ and $\lambda \geq 1$ (observe that the value of $\theta$ can be modified through a different choice of $D$ ). Thus,

$$
\tilde{u}_{\lambda} \geq \lambda^{\frac{1}{1-\theta}} \hat{u}_{1}
$$


for $\lambda \geq 1$. On the other hand, if $\psi_{0}$ is the solution to

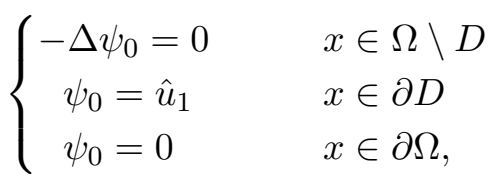

then

$$
\tilde{u}_{\lambda} \geq \lambda^{\frac{1}{1-\theta}} \psi_{0} \quad \text { in } \Omega \backslash D .
$$

Hence, (2.3) holds for $\psi$ defined as $\hat{u}_{1}$ in $D$ and $\psi_{0}$ elsewhere. This concludes the proof.

Our next purpose is to discuss the linearization of problem (1.1) around a possible positive solution $u$. We are therefore assuming that $u \in C^{1}(\bar{\Omega})$, $u(x)>0$ for $x \in \Omega$ together with $u(x)=0$ and $\frac{\partial u}{\partial \nu}(x)<0$ at every $x \in \partial \Omega$. This implies the existence of constants $k, K>0$ such that

$$
k d(x) \leq u(x) \leq K d(x) \quad x \in \Omega .
$$

From now on, the function $d(x)$ will stand for a smooth positive extension of the function $\operatorname{dist}(x, \partial \Omega)$ from a neighborhood of $\partial \Omega$ to the whole of $\Omega$.

Lemma 5. Assume that $q \in C^{\gamma}(\bar{\Omega})$ satisfies $(H)_{q}$. Then, the eigenvalue problem

$$
\left\{\begin{array}{cl}
-\Delta v=\lambda q u^{q(x)-1} v+\sigma v & x \in \Omega \\
v=0 & x \in \partial \Omega,
\end{array}\right.
$$

admits a first eigenvalue $\sigma=\sigma_{1}$ which exhibits the following properties.

i) Every associated weak eigenfunction $\phi \in H_{0}^{1}(\Omega)$ has constant sign in $\Omega$. Moreover, $\phi \in C_{0}^{1}(\bar{\Omega}) \cap C^{2, \alpha}(\Omega)$, for some $0<\alpha<1$.

ii) $\sigma_{1}$ is the unique eigenvalue with a one-signed eigenfunction.

iii) $\sigma_{1}$ is simple.

Proof. To prove the existence, consider the quadratic functional

$$
J_{1}(v)=\frac{1}{2} \int_{\Omega}\left(|\nabla v|^{2}-\lambda q u^{q-1} v^{2}\right),
$$

defined on the manifold $\mathcal{M}=\left\{v \in H_{0}^{1}(\Omega): \int_{\Omega} v^{2}=1\right\}$. Then, $J_{1}$ is coercive on $\mathcal{M}$. In fact,

$$
\begin{aligned}
\int_{\Omega} \lambda q u^{q-1} v^{2} & =\int_{\Omega} \lambda u^{q}\left(\frac{d}{u}\right)\left|\frac{v}{d}\right||v| \\
& \leq C\left\|u^{q}\right\|_{L^{\infty}(\Omega)}\|v\|_{H_{0}^{1}(\Omega)} \\
& \leq C_{\varepsilon}+\varepsilon\|v\|_{H_{0}^{1}(\Omega)}^{2},
\end{aligned}
$$

for every $v \in \mathcal{M}$, where $\varepsilon \in(0,1 / 2)$ and $C_{\varepsilon}>0$. Notice that here we used Hardy's inequality ([3], [6]). Thus,

$$
J_{1}(v) \geq \frac{1}{2}(1-\varepsilon)\|v\|_{H_{0}^{1}(\Omega)}^{2}-\frac{C_{\varepsilon}}{2} \quad v \in \mathcal{M},
$$

and $J_{1}$ is coercive on $\mathcal{M}$. Hence,

$$
\frac{\sigma_{1}}{2}:=\inf _{\mathcal{M}} J_{1}
$$


is achieved at some $\phi \in \mathcal{M}$ that can be chosen nonnegative since $\phi$ may be replaced by $|\phi|$. Therefore

$$
\int_{\Omega} \nabla \phi \nabla v-\lambda u^{q-1} v=\sigma_{1} \int_{\Omega} \phi v
$$

for all $v \in H_{0}^{1}(\Omega)$. Since any other eigenpair $(\sigma, \psi) \in \mathbb{R} \times H_{0}^{1}(\Omega)$ must satisfy (2.8) with $\left(\sigma_{1}, \phi\right)$ replaced by $(\sigma, \psi)$, it is clear that $\sigma_{1}$ is the first (i.e. the smallest) eigenvalue to (2.7). It also holds that $\sigma_{1}$ is the only eigenvalue with a one-signed associated eigenfunction: any eigenfunction $\psi$ of a different eigenvalue $\sigma$ must satisfy $\int_{\Omega} \phi \psi=0$. Finally, if $\phi$ is any nonnegative eigenfunction associated to $\sigma_{1}$ then $\phi(x)>0$ a.e. in $\Omega$. In fact, $-\Delta \phi+M \phi \geq 0$ for some $M>0$ and the strong maximum principle ([24], Theorem 8.19) yields the conclusion.

Let us next show that any other eigenfunction $\tilde{\phi}$ associated to $\sigma_{1}$ is onesigned. In fact, if $\tilde{\phi}_{+} \neq 0$ (here $\tilde{\phi}_{ \pm}=$stands for the positive/negative part of $\tilde{\phi})$ then, by setting $v=\tilde{\phi}_{+}$in $(2.8)$ we get,

$$
\int_{\Omega}\left|\nabla \tilde{\phi}_{+}\right|^{2}-\lambda u^{q-1} \tilde{\phi}_{+}^{2}=\sigma_{1} \int_{\Omega} \tilde{\phi}_{+}^{2} .
$$

This means that $\tilde{\phi}_{+}$is an eigenfunction and so $\tilde{\phi}_{+}>0$. Hence $\tilde{\phi}_{-}=0$ as we wanted to prove.

Let us address now the more subtle regularity issue. If $\phi \in H_{0}^{1}(\Omega)$ is an eigenfunction then, Hardy's inequality implies that $f_{1}(x):=\lambda u^{q-1} \phi+\sigma_{1} \phi \in$ $L^{2}(\Omega)$ and so $\phi \in H^{2}(\Omega)=W^{2,2}(\Omega)$. But applying again Hardy inequality it follows that $\phi / d \in H^{1}(\Omega)$. Hence, $f_{1} \in L^{q_{1}}(\Omega), q_{1}=2^{*}=\frac{2 N}{N-2}$ and so $\phi \in W^{2,2^{*}}(\Omega), \phi / d \in W^{1,2^{*}}(\Omega)$. We thus improve the regularity of the right hand side of 2.7$)$ up to $f_{1} \in L^{q_{2}}(\Omega), q_{2}=q_{1}^{*}=\frac{N q_{1}}{N-q_{1}}$. In general, the right hand side $f_{1}$ improves up to $L^{q_{n}}(\Omega), q_{n}=q_{n-1}^{*}$, provided it belongs to $L^{q_{n-1}}(\Omega)$. This bootstrap argument allows us to assert that $\phi$ is at least in $C_{0}^{1}(\bar{\Omega})$. The $C^{2, \alpha}(\Omega)$ assertion is then a consequence of classical interior regularity since $f_{1} \in C^{\alpha}(\Omega)$.

The simplicity of $\sigma_{1}$ is proved by combining the ideas in 9 and 28]. In fact, let $\phi, \psi$ be two positive eigenfunctions associated to $\sigma_{1}$, fix $\varepsilon>0$ small and set $\phi_{\varepsilon}=\phi+\varepsilon, \psi_{\varepsilon}=\psi+\varepsilon$. Then, integration by parts leads to the equality:

$$
\begin{aligned}
I_{\varepsilon}: & =\int_{\Omega}\left(\nabla \phi_{\varepsilon} \nabla \tilde{\phi}_{\varepsilon}-\nabla \psi_{\varepsilon} \nabla \tilde{\psi}_{\varepsilon}\right) \\
& =\int_{\Omega}\left(\lambda u^{q-1}+\sigma_{1}\right)\left(\frac{\phi}{\phi_{\varepsilon}}-\frac{\psi}{\psi_{\varepsilon}}\right)\left(\phi_{\varepsilon}^{2}-\psi_{\varepsilon}^{2}\right),
\end{aligned}
$$

where the test functions $\tilde{\phi}_{\varepsilon}, \tilde{\psi}_{\varepsilon}$ are defined as

$$
\tilde{\phi}_{\varepsilon}=\frac{\phi_{\varepsilon}^{2}-\psi_{\varepsilon}^{2}}{\phi_{\varepsilon}} \quad \tilde{\psi}_{\varepsilon}=\frac{\phi_{\varepsilon}^{2}-\psi_{\varepsilon}^{2}}{\psi_{\varepsilon}}
$$

It follows that

$$
I_{\varepsilon}=\int_{\Omega}\left|\nabla \phi_{\varepsilon}-\frac{\phi_{\varepsilon}}{\psi_{\varepsilon}} \nabla \psi_{\varepsilon}\right|^{2}+\left|\nabla \psi_{\varepsilon}-\frac{\psi_{\varepsilon}}{\phi_{\varepsilon}} \nabla \phi_{\varepsilon}\right|^{2}
$$


Taking $\liminf \operatorname{in}_{\varepsilon \rightarrow 0} I_{\varepsilon}$ in the second integral in (2.9), using Fatou's Lemma and taking into account 2.10 we obtain,

$$
\int_{\Omega}\left|\nabla \phi-\frac{\phi}{\psi} \nabla \psi\right|^{2}+\left|\nabla \psi-\frac{\psi}{\phi} \nabla \phi\right|^{2}=0 .
$$

Thus, $\psi$ is a scalar multiple of $\phi$. This completes the proof of the lemma.

\section{3. $L^{\infty}$ ESTIMATES}

The purpose of this section is to obtain a priori $L^{\infty}$ bounds for all possible positive solutions of (1.1), with the additional assumption (1.6). These bounds will be required in the proof of iv) in Theorem 1. For technical reasons, we need also that $q>1$ on $\partial \Omega_{+} \cap \Omega$ in case $\Omega_{+}$meets the boundary $\partial \Omega$ (otherwise we have $\bar{\Omega}_{+} \subset \Omega$ ).

Theorem 6. Assume that $q \in C^{\gamma}(\bar{\Omega})$ satisfies $(H)_{q}$ and (1.6), and either $\Omega_{+} \subset \bar{\Omega}_{+} \subset \Omega$ or $q>1$ on $\partial \Omega_{+} \cap \partial \Omega$ in case that the latter set is nonempty. Let $\left\{\left(\lambda_{n}, u_{n}\right)\right\}$ be a family of nonnegative solutions to (1.1) with $\lambda_{n}>0$ varying in a compact interval $I \subset(0, \infty)$. Then, there exists a positive constant $M$ such that:

$$
\left\|u_{n}\right\|_{L^{\infty}(\Omega)} \leq M
$$

for every $n \in \mathbb{N}$. first.

The proof this theorem relies on some lemmas which we state and prove

Lemma 7. Assume $q \in C^{\gamma}(\bar{\Omega})$ satisfies $(H)_{q}$. Let $\left\{\left(\lambda_{n}, u_{n}\right)\right\}$ be a family of weak solutions to

$$
\begin{cases}-\Delta u=\lambda_{n}|u|^{q(x)-1} u & x \in \Omega \\ u=0 & x \in \partial \Omega,\end{cases}
$$

such that $\lambda_{n}>0$ is bounded and

$$
\left\|u_{n}\right\|_{L^{\infty}\left(\Omega_{+}\right)} \leq M
$$

for all $n$ and a certain constant $M>0$. Then, there exists $M_{1}>0$ such that

$$
\left\|u_{n}\right\|_{L^{\infty}(\Omega)} \leq M_{1}
$$

for all $n \in \mathbb{N}$.

Proof. By (3.3) it follows that $u_{n}$ is uniformly bounded on $\partial \Omega_{+}$. Thus, when restricted to $\Omega_{-}, u_{n}$ satisfies

$$
\begin{cases}-\Delta u_{n}=\lambda_{n}\left|u_{n}\right|^{q(x)-1} u_{n} & x \in \Omega_{-} \\ u_{n}=\varphi_{n} & x \in \partial \Omega_{-},\end{cases}
$$

with a family $\varphi_{n}$ of Dirichlet data which are uniformly bounded on $\partial \Omega_{-}$. Since $q<1$ in $\Omega_{-}$this implies that the sequence $u_{n}$ remains uniformly bounded in $\Omega_{-}$, therefore in $\Omega$. This concludes the proof. 
Lemma 8. Let $q \in C^{\gamma}(\bar{\Omega})$ be a positive function in $\bar{\Omega}$ satisfying the growth condition (1.6),

$$
0<q(x)<\frac{N}{N-2} \quad x \in \bar{\Omega} .
$$

Assume that for every $n \in \mathbb{N}, u_{n} \in H_{0}^{1}(\Omega)$ is a weak solution to the problem (3.2), where $\lambda_{n}>0$ is a bounded sequence. If for some $D \subset \subset \Omega$ and some $p>\max \left\{\sup _{\Omega} q, 1\right\}$, we have

$$
\left\|u_{n}\right\|_{L^{p}\left(D_{2 \varepsilon}\right)} \leq M
$$

for all $n$ and a certain $M>0$, where $D_{2 \varepsilon}=B(D, 2 \varepsilon)$, then $\left\|u_{n}\right\|_{L^{\infty}\left(D_{\varepsilon}\right)}$ is also bounded.

Proof. Choose a family of domains $D_{k}$ verifying $D_{\varepsilon} \subset D_{k} \subset D_{2 \varepsilon}$ and $\overline{D_{k+1}} \subset$ $D_{k}$. Taking $\gamma>1$ such that

$$
\sup _{\Omega} q \leq \gamma<\frac{N}{N-2},
$$

together with $\gamma<p$, we observe that if $f(x, u)=|u|^{q(x)-1} u$ then

$$
\left|\lambda_{n} f(x, u)\right| \leq C\left(1+|u|^{\gamma}\right),
$$

for $x \in \Omega$ and $u \in \mathbb{R}$. Keeping the notation of Lemma 3 , for a function $u$ defined in $\Omega$ we set $N_{f}(u)(\cdot)=f(\cdot, u(\cdot))$.

Suppose that $\left\|u_{n}\right\|_{L^{p_{k}\left(D_{k}\right)}}$ is bounded for some $k \geq 1$ and a certain $p_{k}>\gamma$. Then,

$$
\left\|N_{f}\left(u_{n}\right)\right\|_{L^{r}\left(D_{k}\right)} \leq C,
$$

for some $C>0$, with $r_{k}=p_{k} / \gamma$. By using interior $W^{2, r_{k}}$ estimates and the Sobolev embedding we find that

$$
\left\|u_{n}\right\|_{L^{p_{k+1}\left(D_{k+1}\right)}} \leq C,
$$

for a certain constant $C$ and

$$
p_{k+1}=\frac{N r_{k}}{N-2 r_{k}} .
$$

Starting the iteration with $p_{1}=p$ and observing that

$$
\frac{p_{k+1}}{p_{k}} \geq 1+\delta,
$$

with

$$
\delta=\frac{1}{\gamma} \frac{N}{N-2}-1,
$$

we achieve a bound for $u_{n}$ in $L^{\infty}\left(D_{\varepsilon}\right)$ after finitely many steps.

Lemma 9. Suppose that $q \in C^{\gamma}(\bar{\Omega})$ satisfies $(H)_{q}$ and $(1.6)$ and let $Q \subset \Omega_{+}$ be an open subset such that

$$
Q \subset \bar{Q} \subset \Omega .
$$

Let $\left\{\left(\lambda_{n}, u_{n}\right)\right\}$ be a sequence of weak solutions to (3.2) with $\lambda_{n}>0$ bounded, $u_{n}$ positive and

$$
\left\|u_{n}\right\|_{L^{\infty}(Q)} \rightarrow \infty .
$$

Then, there exits $x_{0} \in Q$ such that

$$
\lim _{n} u_{n}\left(x_{0}\right)=\infty .
$$


Proof. A small $\varepsilon>0$ can be found such that

$$
\bar{Q} \subset D:=B_{1} \cup \cdots \cup B_{M},
$$

where $B_{i}=B_{i}\left(x_{i}, \varepsilon\right), 1 \leq i \leq M$, is an open ball with center $x_{i} \in Q$ such that $\bar{B}_{i}\left(x_{i}, 2 \varepsilon\right) \subset \Omega$. Since $\left\|u_{n}\right\|_{L^{\infty}\left(Q_{2 \varepsilon}\right)} \rightarrow \infty$, by Lemma 8 , an exponent $p>1$ can be found so that

$$
\sup _{\Omega} q<p<\frac{N}{N-2}
$$

together with

$$
\lim _{n}\left\|u_{n}\right\|_{L^{p}(D)}=\infty .
$$

Thus, there exists $1 \leq i \leq M$ satisfying,

$$
\lim _{n}\left\|u_{n}\right\|_{L^{p}\left(B_{i}\right)}=\infty \text {. }
$$

Now, Theorem 8.18 in [24] implies that

$$
\left\|u_{n}\right\|_{L^{p}\left(B_{i}\right)} \leq \frac{C}{\left(\frac{N}{N-2}-p\right)^{2}} \inf _{\frac{1}{2} B_{i}} u_{n} \rightarrow \infty,
$$

for a certain $C>0$, where $\frac{1}{2} B_{i}=B\left(x_{i}, \frac{\varepsilon}{2}\right)$. The existence of $x_{0} \in Q$ satisfying (3.6) is therefore shown.

We finally consider a result which will be used when obtaining local bounds for scaled solutions in the proof of Theorem 6 .

Lemma 10. Assume that $u>0$ is a weak solution to

$$
-\Delta u=\lambda a(x) u^{q(x)},
$$

in a ball $B_{4 R}$ with radius $4 R, a \in L^{\infty}\left(B_{4 R}\right), a(x) \geq a_{0}>0, \lambda$ varies in a compact interval $I \subset(0, \infty)$, and the exponent $q$ satisfies,

$$
1<q_{0} \leq q(x) \leq q_{1} \quad x \in B_{4 R} .
$$

Then, a positive constant $C=C(R, q, N)$ exists such that the following Harnack inequality holds:

$$
\sup _{B_{R}} u \leq C \inf _{B_{R}} u,
$$

where $B_{R}$ is the ball concentric with $B_{4 R}$ and radius $R$.

For the proof of Lemma 10, we need an auxiliary estimate for solutions, which we state and prove first.

Lemma 11. Assume that $u>0$ is a weak solution to the inequality

$$
-\Delta u \geq \lambda a(x) u^{q(x)}
$$

in a ball $B_{2 R}$ with radius $2 R, a \in L^{\infty}\left(B_{3 R}\right), a(x) \geq a_{0}>0$, and

$$
q(x) \geq q_{0}>1 .
$$

Then, for all $\gamma>q_{0}-1$ there exists a constant $C>0$ such that

$$
\int_{B_{R}} u^{\gamma} \leq C\left(R^{N}+R^{N-\frac{2 \gamma}{q_{0}-1}}\right)
$$


where $C$ can be taken independent of $\lambda$ varying in a compact interval $I \subset$ $(0, \infty)$.

Proof. We assume the center of $B_{2 R}$ to be the origin. Suppose also $\lambda_{0} \leq$ $\lambda \leq \lambda_{1}$. A function $\xi \in C_{0}^{\infty}(B(0,2)), 0 \leq \xi \leq 1$ is chosen so that $\xi \equiv 1$ in $\overline{B(0,1)},|\nabla \xi| \leq 2$.

For $k$ to be determined, the test function

$$
\phi(x)=\xi\left(\frac{x}{R}\right)^{k} u(x)^{-\theta} \quad \theta=q_{0}-\gamma,
$$

is introduced in the weak differential inequality (3.8) to obtain,

$$
\lambda_{0} a_{0} \int_{B_{2 R}} u^{q(x)} \phi \leq \int_{B_{2 R}} \nabla u \nabla \phi
$$

and so,

$$
\int_{B_{2 R}} \theta \xi^{k} u^{-\theta-1}|\nabla u|^{2}+\lambda_{0} a_{0} \xi^{k} u^{q(x)-\theta} \leq \frac{2 k}{R} \int_{B_{2 R}} \xi^{k-1} u^{-\theta}|\nabla u| .
$$

Young's inequality allows us to write the estimate

$$
\xi^{k-1} u^{-\theta}|\nabla u| \leq \frac{\theta R}{4 k} \xi^{k} u^{-\theta-1}|\nabla u|^{2}+\frac{k}{\theta R} \xi^{k-2} u^{-\theta+1} .
$$

Thus,

$$
\int_{B_{2 R}} \frac{\theta}{2} \xi^{k} u^{-\theta-1}|\nabla u|^{2}+\lambda_{0} a_{0} \int_{B_{2 R}} \xi^{k} u^{q(x)-\theta} \leq \frac{2 k^{2}}{\theta R^{2}} \int_{B_{2 R}} \xi^{k-2} u^{-\theta+1} .
$$

In particular,

$$
\int_{B_{2 R}} \xi^{k} u^{q(x)-\theta} \leq \frac{C}{R^{2}} \int_{B_{2 R}} \xi^{k-2} u^{-\theta+1},
$$

for a certain positive constant $C$.

We now observe that,

$$
\int_{B_{2 R}} \xi^{k} u^{q(x)-\theta} \geq \int_{B_{2 R} \cap\{u \geq 1\}} \xi^{k} u^{q_{0}-\theta}=\int_{B_{2 R} \cap\{u \geq 1\}} \xi^{k} u^{\gamma} .
$$

Fixing $k$ and $p$ so that $(1-\theta) p=\gamma$ and $p(k-2)=k$, Young's inequality implies that

$$
\frac{1}{R^{2}} \xi^{k-2} u^{1-\theta} \leq \frac{\varepsilon^{p}}{p} \xi^{k} u^{\gamma}+\frac{1}{p^{\prime} \varepsilon^{p^{\prime}} R^{2 p^{\prime}}}
$$

for every $\varepsilon>0$, with $p=\frac{\gamma}{1-\theta}$ and $p^{\prime}=\frac{\gamma}{q_{0}-1}$. A suitable choice of $\varepsilon>0$ gives,

$$
\frac{C}{R^{2}} \int_{B_{2 R}} \xi^{k-2} u^{-\theta+1} \leq \frac{1}{3} \int_{B_{2 R}} \xi^{k} u^{\gamma}+C R^{N-\frac{2 \gamma}{q_{0}-1}} .
$$

Combining (3.9), (3.10) and (3.11) we obtain

$$
\int_{B_{2 R} \cap\{u \geq 1\}} \xi^{k} u^{\gamma} \leq \frac{1}{3} \int_{B_{2 R} \cap\{u \geq 1\}} \xi^{k} u^{\gamma}+\frac{1}{3} \int_{B_{2 R} \cap\{u<1\}} \xi^{k} u^{\gamma}+C R^{N-\frac{2 \gamma}{q_{0}-1}} .
$$

Thus,

$$
\int_{B_{2 R} \cap\{u \geq 1\}} \xi^{k} u^{\gamma} \leq C R^{N}+C R^{N-\frac{2 \gamma}{q_{0}-1}} .
$$

The desired inequality follows from this estimate. 
Proof of Lemma 10. The assertion in the lemma is a consequence of Lemma 4.2 in [39] (see also Theorem 5 in [38) according to the following arguments: the equation satisfied by $u$ has the form,

$$
-\Delta u=f(x, u),
$$

where $|f(x, u)| \leq d(x)|u|$, with $d=\lambda|a(x)||u|^{q(x)-1}$. We are showing that for every $p \in\left(\frac{N}{2}, N\right)$ the norm $\|d\|_{L^{p}\left(B_{2 R}\right)}$ can be estimated solely in terms of $R, N$ and $q$. Therefore, the above mentioned result in [39] ensures the existence of a constant $C$ depending on $N, q$ and $R^{2-\frac{N}{p}}\|d\|_{L^{p}\left(B_{2 R}\right)}$ such that (3.7) holds.

To show the assertion on the estimate of $\|d\|_{L^{p}\left(B_{2 R}\right)}$ let $\lambda=\|a\|_{L^{\infty}(\Omega)}=1$ for simplicity, and observe that

$$
\begin{aligned}
\|d\|_{L^{p}\left(B_{2 R}\right)}^{p} & =\int_{B_{2 R}} u^{p(q(x)-1)} \leq \int_{B_{2 R} \cap\{u>1\}} u^{p\left(q_{1}-1\right)}+C R^{N} \\
& \leq \int_{B_{2 R}} u^{p\left(q_{1}-1\right)}+C R^{N} .
\end{aligned}
$$

Since $\gamma:=p\left(q_{1}-1\right)>q_{0}-1$ for every $p>N / 2$ then Lemma 11 implies that

$$
\int_{B_{2 R}} u^{\gamma} \leq C\left(R^{N}+R^{N-\frac{2 \gamma}{q_{0}-1}}\right)
$$

for a certain positive constant $C>0$. Therefore, the proof is concluded.

We end this section with the proof of our main result, Theorem 6 .

Proof of Theorem 6. If (3.1) fails then Lemma 7 implies that

$$
\lim _{n}\left\|u_{n}\right\|_{L^{\infty}\left(\Omega_{+}\right)}=\infty \text {. }
$$

Accordingly, a sequence $x_{n} \in \bar{\Omega}_{+}$exists so that

$$
\lim _{n} u_{n}\left(x_{n}\right)=\infty \text {. }
$$

After passing to a subsequence if necessary there exist $\lambda_{0}>0$ and $x_{0} \in \bar{\Omega}_{+}$ verifying $\lambda_{n} \rightarrow \lambda_{0}$ and $x_{n} \rightarrow x_{0}$. Now, three options are possible:

$$
\begin{aligned}
& \text { (1) } x_{0} \in \Omega_{+} . \\
& \text {(2) } x_{0} \in \partial \Omega_{+} \cap \partial \Omega . \\
& \text { (3) } x_{0} \in \Gamma_{+}=\partial \Omega_{+} \cap \Omega .
\end{aligned}
$$

As can be checked next, cases (1) and (2) can be handled according to the general arguments in [23]. However, case (3) is quite less standard and will be separately treated later.

To proceed in case (1) we set (see [23]):

$$
v_{n}(y)=\frac{1}{M_{n}} u_{n}\left(x_{n}+\theta_{n} y\right)
$$

where we assumed that $u_{n}\left(x_{n}\right)=\sup _{\Omega_{+}} u_{n}=: M_{n}$ and choose $\theta_{n}=1 / M_{n}^{\frac{q\left(x_{n}\right)-1}{2}}$. Then, $v_{n}$ satisfies

$$
-\Delta v_{n}=\lambda_{n} M_{n}^{\Delta_{n} q} v_{n}^{q\left(x_{n}+\theta_{n} y\right)}
$$


with $\Delta_{n} q:=q\left(x_{n}+\theta_{n} y\right)-q\left(x_{n}\right)$, where we are assuming that

$$
|y|<R_{n}:=\frac{\varepsilon-\left|x_{n}-x_{0}\right|}{\theta_{n}}
$$

and it is supposed that $\bar{B}\left(x_{0}, \varepsilon\right) \subset \Omega$. Observe that, since $q\left(x_{n}\right) \rightarrow q\left(x_{0}\right)>1$ then $\theta_{n} \rightarrow 0$ meanwhile $R_{n} \rightarrow \infty$.

On the other hand,

$$
\left|\Delta_{n} q\right| \leq C R \theta_{n}^{\gamma}
$$

for $|y| \leq R$ and a certain constant $C>0$. This means that, as a function of $y, M_{n}^{\Delta_{n} q} \rightarrow 1$ uniformly on compact sets of $\mathbb{R}^{N}$. Now we fix $R>0$. Let $B_{R}=B(0, R)$, take $n$ large and observe that

$$
\left\|v_{n}\right\|_{L^{\infty}\left(B_{R}\right)} \leq 1
$$

allow us to obtain, via the standard $L^{p}$ estimates, a uniform bound of the form,

$$
\left\|v_{n}\right\|_{C^{1, \beta}\left(\bar{B}_{R}\right)} \leq M,
$$

for a certain positive $M$. This furnishes a subsequence converging in $C^{1}\left(\bar{B}_{R}\right)$ to a nonnegative function $v$ satisfying

$$
-\Delta v=\lambda_{0} v^{q\left(x_{0}\right)},
$$

in both weak and classical senses in $B_{R}$. A further diagonal procedure leads to a nonnegative solution $v$ of such equation in $\mathbb{R}^{N}$ fulfilling $v(0)=1$. Since this is not possible (see [22]), case (1) can be ruled out.

As for case (2) we also proceed following [23]. The argument there is next reviewed for the sake of completeness. By means of a translation and a rotation it can be assumed that $x_{0}=0$ and that $U \cap \Omega=\left\{\left(x^{\prime}, x_{N}\right)\right.$ : $\left.x_{N}>\varphi\left(x^{\prime}\right)\right\}$ with $U=\left[B(0, \eta) \cap\left\{x_{N}=0\right\}\right] \times(-\delta, \delta)$ a neighborhood of $x=0, x^{\prime}=\left(x_{1}, \ldots, x_{N-1}\right)$, and the function $\varphi \in C^{1}\left(B(0, \eta) \cap\left\{x_{N}=0\right\}\right)$ satisfies $\varphi(0)=0, \nabla \varphi(0)=0$. The mapping $x \mapsto y=\left(y^{\prime}, y_{N}\right)$ with $y^{\prime}=x^{\prime}$, $y_{N}=x_{N}-\varphi\left(x^{\prime}\right)$ transforms the equation in (1.1) in:

$$
\begin{aligned}
& -\Delta_{y^{\prime}} u+\left(1+\left|\nabla \varphi\left(y^{\prime}\right)\right|^{2}\right) \partial_{y_{N} y_{N}}^{2} u- \\
& \quad \sum_{i=1}^{N-1} \partial_{y_{i}} \varphi\left(y^{\prime}\right) \partial_{y_{i} y_{N}}^{2} u+\Delta \varphi\left(y^{\prime}\right) \partial_{y_{N}} u=\lambda u^{q(y),}
\end{aligned}
$$

where $y \in V:=\left\{y: y^{\prime} \in\left[B(0, \eta) \cap\left\{y_{N}=0\right\}\right], 0<y_{N}<\delta-\varphi\left(y^{\prime}\right)\right\}$ and $u$ is now regarded as a function of $y$. By setting $y_{n}=\left(x_{n}^{\prime},\left(x_{n}\right)_{N}-\varphi\left(x_{n}^{\prime}\right)\right)$, $d_{n}=\left(x_{n}\right)_{N}-\varphi\left(x_{n}^{\prime}\right)$ and performing the scaling:

$$
v_{n}(z)=\frac{1}{M_{n}} u\left(y_{n}+\theta_{n} z\right),
$$

with $\theta_{n}=1 / M_{n}^{\frac{q\left(y_{n}\right)-1}{2}}, 3.12$ is transformed in:

$$
\begin{array}{r}
-\Delta_{z^{\prime}} v+\left(1+\left|\nabla \varphi\left(y_{n}^{\prime}+\theta_{n} z^{\prime}\right)\right|^{2}\right) \partial_{z_{N} z_{N}}^{2} v-\sum_{i=1}^{N-1} \partial_{y_{i}} \varphi\left(y_{n}^{\prime}+\theta_{n} z^{\prime}\right) \partial_{z_{i} z_{N}}^{2} v \\
+\theta_{n} \Delta \varphi\left(y_{n}^{\prime}+\theta_{n} z^{\prime}\right) \partial_{z_{N}} v=\lambda_{n} M_{n}^{\Delta_{n} q} v^{q\left(y_{n}+\theta_{n} z\right)}
\end{array}
$$


where $z \in \frac{1}{\theta_{n}} V_{n}, V_{n}:=V-y_{n}$ and $\Delta_{n} q=q\left(y_{n}+\theta_{n} z\right)-q\left(y_{n}\right)$. Since we are assuming that $y_{n} \rightarrow 0$ together with $q(0)>1$ then we conclude, as in case (1), that

$$
M_{n}^{\Delta_{n} q} \rightarrow 1
$$

Observe that

$$
v_{n}(z)=0,
$$

for $z_{N}=-d_{n} / \theta_{n}$. Thus, by arguing as in [23], it can be shown that

$$
s:=\frac{\lim }{n} \frac{d_{n}}{\theta_{n}}>0 .
$$

Two options are now possible: $s=\infty$ and $s<\infty$. In the former case and just as in (1) we obtain by means of a suitable limit process a nontrivial nonnegative solution to the equation

$$
-\Delta v=\lambda_{0} v^{q(0)}
$$

in $\mathbb{R}^{N}$, which is verified to be not possible as before. In case $s<\infty$, by using the $L^{p}$ estimates up to the boundary we obtain a nontrivial nonnegative solution to the problem:

$$
\left\{\begin{array}{cl}
-\Delta v=\lambda_{0} v^{q(0)} & z_{N}>-s \\
v=0 & z_{N}=-s .
\end{array}\right.
$$

As proved in 23] this is also impossible. Therefore, case (2) can be discarded.

In the discussion of the final case (3), the approach in 23] can not be employed since, as $q\left(x_{0}\right)=1$, the asymptotic behavior of $M_{n}^{\Delta_{n} q}$ can not be properly determined. We are following instead the ideas developed in [36] (see [2] for a related approach).

As a first remark observe that it can be assumed that

$$
\lim _{n}\left\|u_{n}\right\|_{L^{\infty}(Q)}=\infty
$$

where $Q=\left\{x \in \Omega_{+}: \operatorname{dist}\left(x, \partial \Omega_{+} \cap \partial \Omega\right)>\delta\right\}$, for some $\delta>0$. Otherwise we can apply case (2) which has been already ruled out. Thus, by Lemma 9 we can find some $x_{0} \in Q$ such that $\lim _{n} u_{n}\left(x_{0}\right)=\infty$.

We introduce now the alternative scaling,

$$
v_{n}(y)=\frac{1}{u_{n}\left(x_{0}\right)} u_{n}\left(x_{0}+\theta_{n} y\right)
$$

where $y \in \frac{1}{\theta_{n}} B(0, \eta), \eta>0$ being chosen so that $\bar{B}\left(x_{0}, \eta\right) \subset \Omega_{+}$. By setting $\theta_{n}^{2} u_{n}^{q\left(x_{0}\right)-1}=1$ then $v=v_{n}(y)$ solves the equation:

$$
-\Delta v=\lambda_{n} u_{n}\left(x_{0}\right)^{q\left(x_{0}+\theta_{n} y\right)-q\left(x_{0}\right)} v^{q\left(x_{0}+\theta_{n} y\right)} .
$$

In addition (see the proof of case (1D),

$$
\lim _{n} u_{n}\left(x_{0}\right)^{q\left(x_{0}+\theta_{n} y\right)-q\left(x_{0}\right)}=1,
$$

uniformly on compact sets of $\mathbb{R}^{N}$. 
Fixing $R>0$ we next observe equation (3.14) in $B(0, R)$. Since $q\left(x_{0}+\right.$ $\left.\theta_{n} y\right) \rightarrow q\left(x_{0}\right)>1$ uniformly in $B(0, R)$ then $1<q_{0} \leq q\left(x_{0}+\theta_{n} y\right) \leq q_{1}$ for suitable $q_{0}, q_{1}$. In view of (3.15), Lemma 10 implies

$$
\sup _{B_{\frac{R}{4}}} v_{n} \leq C \inf _{\frac{R}{4}} v_{n} \leq C,
$$

for large $n$, since $\inf _{B_{\frac{R}{4}}} v_{n} \leq 1$. Thus we obtain a bound for $\left\|v_{n}\right\|_{L^{\infty}\left(B_{\frac{R}{4}}\right)}$ and passing through a subsequence we conclude that $v_{n} \rightarrow v$ in $C^{1}\left(\bar{B}_{\frac{R}{4}}^{4}\right)$ being $v$ a weak, and hence a classical nonnegative solution to the equation

$$
-\Delta v=\lambda_{0} v^{q\left(x_{0}\right)} \quad x \in B_{\frac{R}{4}}
$$

satisfying $v(0)=1$. Moreover, by a diagonal procedure, a subsequence $v_{n}$ can be extracted such that $v_{n} \rightarrow v$ in $C^{1}\left(\mathbb{R}^{N}\right), v$ nonnegative, $v(0)=1$ and $v$ solving the previous equation in the whole $\mathbb{R}^{N}$. This is not possible, as before, and the proof of Theorem 6 is finished.

\section{Proof of theorem 1}

Proof of Theorem 1. First, we prove that there exists $\lambda^{* *}>0$ such that no positive solutions to (1.1) are possible when $\lambda \geq \lambda^{* *}$.

To begin with, assume $u \in H_{0}^{1}(\Omega), u \neq 0$, is any nonnegative weak solution to (1.1). Then, Lemma 3 implies that $u$ becomes a classical positive solution $u \in C^{2}, \alpha(\bar{\Omega})$. In addition $\bar{u}=u$ defines a strict supersolution to the auxiliary problem (2.2). As noticed in the proof of Theorem 4 , this problem admits subsolutions as small as desired, and so a particular one $\underline{u}$ can be found so that $\underline{u} \leq \bar{u}$. Therefore, by the method of sub and supersolutions and the uniqueness of the positive solution to 2.2 we obtain

$$
u \geq \tilde{u}_{\lambda} .
$$

Take now an arbitrary subdomain $D \subset \Omega_{+}$having a first Dirichlet eigenvalue $\lambda_{1, D}$ with an associated positive eigenfunction $\phi_{1, D}$. We will show that no positive solutions are possible if $\lambda \geq \lambda^{* *}:=\max \left\{\lambda_{0}, \lambda_{1, D}\right\}$ where $\lambda_{0}$ is fixed so that

$$
\tilde{u}_{\lambda}(x) \geq 1 \quad x \in D,
$$

when $\lambda \geq \lambda_{0}$ (recall that $\tilde{u}_{\lambda} \rightarrow+\infty$ uniformly in compact sets of $\Omega$ by Theorem 4, iii)). In fact, multiplying the equation in (1.1) by $\phi_{1, D}$ and integrating by parts we find that

$$
\lambda \int_{D} \phi_{1, D} u^{q(x)} \leq \lambda_{1, D} \int_{D} \phi_{1, D} u .
$$

If $\lambda \geq \lambda_{0}$ then $u \geq \tilde{u}_{\lambda_{0}} \geq 1$ in $D$. Thus $u<u^{q(x)}$ in $D$ and the previous inequality implies that:

$$
\lambda<\lambda_{1, D}
$$

Therefore, (1.1) does not admit positive solutions if $\lambda \geq \lambda^{* *}$.

To characterize the existence regime of positive solutions we set, as customary,

$$
\mathcal{S}=\{\lambda>0: \text { a positive solution to } 1.1 \text { exists }\}
$$


and define $\lambda^{*}=\sup \mathcal{S}$. To see that the set $\mathcal{S}$ is nonempty, we take $\bar{u}=M \psi$ where $\psi$ is the solution to the problem $-\Delta \psi=1$ in $\Omega, \psi=0$ on $\partial \Omega$, and $M$ is a positive constant. It is not hard to see that $\bar{u}$ becomes a supersolution to (1.1) if $\lambda$ is small enough to have $M^{1-q(x)} \geq \lambda$ in $\Omega$. A comparable subsolution $\underline{u}$ is obtained by setting $\underline{u}=\tilde{u}_{\lambda^{\prime}}$ with $0<\lambda^{\prime} \leq \lambda$ small enough. As a byproduct, we obtain that $[0, \lambda] \subset \mathcal{S}$, and in particular $0<\lambda^{*}<\infty$. A similar argument shows that actually $\mathcal{S}=\left[0, \lambda^{*}\right)$ or $\mathcal{S}=\left[0, \lambda^{*}\right]$. Thus it is clear that a positive solution to (1.1) exists if $0<\lambda<\lambda^{*}$ while solutions do not exist if $\lambda>\lambda^{*}$.

On the other hand, notice that, by (4.1), since $\tilde{u}_{\lambda}$ lies below any positive solution to (1.1) it is well-known that there exists a minimal positive solution for every $\lambda \in\left(0, \lambda^{*}\right)$.

Let us prove now the monotonicity of $u_{\lambda}$ in $\lambda$. In fact if $\lambda<\lambda^{\prime}$ then $\bar{u}=u_{\lambda^{\prime}}$ defines a supersolution to (1.1) while $\underline{u}=\tilde{u}_{\lambda}$ defines a subsolution to that problem with $\underline{u} \leq \bar{u}$. Therefore $\tilde{u}_{\lambda}<u_{\lambda}<u_{\lambda^{\prime}}$ in $\Omega$, as was wanted to prove.

Consider next any family $v_{\lambda}$ of positive solutions such that

$$
\left\|v_{\lambda}\right\|_{L^{\infty}(\Omega)}=O(1) \quad \text { as } \lambda \rightarrow 0 .
$$

Choosing a sequence $v_{\lambda_{n}}$ with $\lambda_{n} \rightarrow 0$ and using $L^{p}$ and Schauder estimates we conclude that, modulus a subsequence, $v_{\lambda_{n}} \rightarrow 0$ in, say, $C^{2}(\bar{\Omega})$ (observe that $v=0$ is the only solution to (1.1) if $\lambda=0$ ). Thus, the whole family $v_{\lambda} \rightarrow 0$ in $C^{2}(\bar{\Omega})$. In particular, $v=v_{\lambda}$ satisfies

$$
-\Delta v \leq \lambda v^{q_{-}} \quad x \in \Omega
$$

for small $\lambda$ and so

$$
v_{\lambda} \leq \lambda^{\frac{1}{1-q_{-}}} \varphi(x),
$$

for small $\lambda$, where $q_{-}=\inf _{\Omega} q$ and $\varphi$ is the positive solution to $-\Delta \varphi=\varphi^{q_{-}}$ in $\Omega$ together with $\varphi_{\mid \partial \Omega}=0$.

Concerning the continuity assertion suppose $\lambda_{n} \rightarrow \bar{\lambda}$ in $\left(0, \lambda^{*}\right)$ with $\lambda_{n}$ increasing. Since $u_{n}:=u_{\lambda_{n}} \leq u_{\bar{\lambda}}$ and $u_{\bar{\lambda}} \in L^{\infty}(\Omega)$ we first obtain a $C^{1, \beta}(\bar{\Omega})$ uniform estimate for $u_{n}$ and thus, we achieve a uniform estimate in $C^{2, \alpha}(\bar{\Omega})$. Therefore, $u_{n} \rightarrow \hat{u}$ in $C^{2}(\bar{\Omega})$ where $\hat{u} \leq u_{\bar{\lambda}}$ solves (1.1) at $\lambda=\bar{\lambda}$. Since $u_{\bar{\lambda}}$ is the minimal solution then $\hat{u}=u_{\bar{\lambda}}$ and we are done. This completes the proofs of i) and ii).

To show iii) we follow the argument in 32 and set $\mathcal{K}=\left\{u \in H_{0}^{1}(\Omega)\right.$ : $\left.\tilde{u}_{\lambda} \leq u \leq u_{\lambda}\right\}$. Then, the functional

$$
J(u)=\int_{\Omega}\left(\frac{1}{2}|\nabla u|^{2}-\frac{\lambda}{q+1}|u|^{q+1}\right),
$$

achieves a minimum $\hat{u}$ in $\mathcal{K}$ which turns out to be a solution to 1.1) (see [41]). Thus, $\hat{u}=u_{\lambda}$.

Choose now $\varphi \in C_{0}^{1}(\bar{\Omega})$ nonnegative. Then,

$$
D^{2} J\left(u_{\lambda}\right)[\varphi]=\int_{\Omega}|\nabla \varphi|^{2}-\lambda q u_{\lambda}^{q-1} \varphi^{2} \geq 0 .
$$

Since an arbitrary nonnegative $\varphi \in H_{0}^{1}(\Omega)$ can be approached in $H_{0}^{1}(\Omega)$ by a sequence of nonnegative functions $\varphi_{n} \in C_{0}^{1}(\bar{\Omega})$, then 4.2 holds for 
nonnegative functions in $H_{0}^{1}(\Omega)$. Moreover, for arbitrary $\varphi \in H_{0}^{1}(\Omega)$, writing $\varphi=\varphi_{+}-\varphi_{-}$, we have

$$
D^{2} J\left(u_{\lambda}\right)[\varphi]=D^{2} J\left(u_{\lambda}\right)\left[\varphi_{+}\right]+D^{2} J\left(u_{\lambda}\right)\left[\varphi_{-}\right] \geq 0 .
$$

This proves iii).

The proof of iv) involves a topological degree argument. To settle the abstract framework we set $X=C_{0}^{1}(\bar{\Omega})=\left\{u \in C^{1}(\bar{\Omega}): u_{\mid \partial \Omega}=0\right\}$, and, denoting as usual the dual space of $H_{0}^{1}(\Omega)$ as $H^{-1}(\Omega)$, we define the solution operator $G: H^{-1}(\Omega) \rightarrow H_{0}^{1}(\Omega)$ as the continuous (linear) operator mapping $g \in H^{-1}(\Omega)$, to the weak solution $u \in H_{0}^{1}(\Omega)$ of the problem $-\Delta u=g$ in $\Omega, u_{\mid \partial \Omega}=0$. By $N_{f}: L^{\infty}(\Omega) \rightarrow L^{\infty}(\Omega)$ we denote the Nemytskii operator associated to $f(x, u)=|u|^{q(x)-1} u$, mapping $u$ to the function $|u|^{q(\cdot)-1} u$ (Lemma 3). Then, the operator

$$
\begin{aligned}
H:[0, \infty) \times X & \longrightarrow X \\
(\lambda, u) & \longmapsto H(\lambda, u)=\lambda G\left(N_{f}(u)\right),
\end{aligned}
$$

exhibits the following features:

(1) $H$ is completely continuous, i.e., is continuous and maps bounded sets into relatively compact sets of $X$.

(2) $H$ is strongly order preserving, i.e., $u \leq v$ and $u \neq v$ implies $\tilde{u}(x)<$ $\tilde{v}(x)$ for $x \in \Omega$ and $\frac{\partial \tilde{u}}{\partial \nu}(x)>\frac{\partial \tilde{v}}{\partial \nu}(x)$ for each $x \in \partial \Omega$, where $\tilde{u}=$ $H(\lambda, u(\cdot)), \tilde{v}=H(\lambda, v(\cdot))$.

(3) A function $u \in X$ positive in $\Omega$ defines a weak solution to (1.1) if and only if $u=H(\lambda, u)$.

A proof of the previous assertions is more or less standard and is therefore omitted.

Now fix $0<\bar{\lambda}_{1}<\lambda^{*}$. We already know that problem (1.1) admits a minimal solution $u_{1}:=u_{\bar{\lambda}_{1}}$ and we are looking for a second positive solution. To this end we choose $0<\underline{\lambda}<\bar{\lambda}_{1}<\bar{\lambda}<\lambda^{*}$, set $\underline{u}=u_{\underline{\lambda}}, \bar{u}=u_{\bar{\lambda}}$ and observe that $u_{1} \in \mathcal{C}:=\{u \in X: \underline{u}(x) \leq u(x) \leq \bar{u}(x), x \in \Omega\}$. Moreover, the strong maximum principle ([24]) implies that

$$
H_{\bar{\lambda}_{1}}(\mathcal{C}) \subset \operatorname{int}(\mathcal{C})
$$

where for $\lambda>0, H_{\lambda}: X \rightarrow X$ stands for the operator mapping $u$ to the function $H(\lambda, u(\cdot))$, and $\operatorname{int}(\mathcal{C})$ designates the interior of $\mathcal{C}$ relative to the topology of $X$. In particular, a ball $B_{\varepsilon}:=B\left(u_{1}, \varepsilon\right)$ in $X, \varepsilon>0$ small, can be found so that $B_{\varepsilon} \subset \bar{B}_{\varepsilon} \subset \mathcal{C}$.

The proof of existence of an additional positive solution to (1.1), $\lambda=\bar{\lambda}_{1}$, is obtained by reaching a contradiction if it is assumed that $u_{1}$ is the unique positive solution. In fact, under the latter assumption the Leray-Schauder degree

$$
d\left(I-H_{\bar{\lambda}_{1}}, B_{\varepsilon}, 0\right)
$$

is well defined. To compute its value we observe that the fixed point index $i\left(H_{\bar{\lambda}_{1}}, \mathcal{C}, \mathcal{C}\right)$ of $H_{\bar{\lambda}_{1}}$ relative to the closed and convex set $\mathcal{C}$ is well defined while

$$
i\left(H_{\bar{\lambda}_{1}}, \mathcal{C}, \mathcal{C}\right)=1
$$


(see [1], Section 11). The isolation of $u_{1}$ together with the excision property of the index ([1]) implies that,

$$
i\left(H_{\bar{\lambda}_{1}}, \mathcal{C}, \mathcal{C}\right)=i\left(H_{\bar{\lambda}_{1}}, B_{\varepsilon}, \mathcal{C}\right) .
$$

Since $\mathcal{C}$ is a retract of the whole space $X$, a retraction $r: X \rightarrow \mathcal{C}$ exists such that the restriction $r_{\mathcal{C}}$ is the identity in $\mathcal{C}$. Accordingly, $r(u)=u$ for all $u \in B_{\varepsilon}$. Moreover, $r^{-1}\left(B_{\varepsilon}\right)=B_{\varepsilon}$. Thus, the definition of the fixed point index in terms of the Leray-Schauder degree ([1]) permits us writing,

$$
i\left(H_{\bar{\lambda}_{1}}, B_{\varepsilon}, \mathcal{C}\right)=d\left(I-H_{\bar{\lambda}_{1}}, r^{-1}\left(B_{\varepsilon}\right), 0\right)=d\left(I-H_{\bar{\lambda}_{1}}, B_{\varepsilon}, 0\right) .
$$

Thus (see Remark 3 below),

$$
d\left(I-H_{\bar{\lambda}_{1}}, B_{\varepsilon}, 0\right)=1 .
$$

We introduce now the open set $Q$ in $X$ given by,

$$
Q=\{u \in X: \bar{u}(x)-\delta<u(x)<M+1 \text { for all } x \in \Omega\},
$$

where $M$ is a uniform $L^{\infty}$ estimate of the positive solutions to (1.1) corresponding to the interval of the parameter $I=\left[\bar{\lambda}_{1}, \lambda^{*}+1\right]$ (Theorem 6 ). Since no positive solutions $u$ to 1.1 on the boundary $\partial Q$ exist for all $\lambda \in I$, then the Leray-Schauder degree $d\left(I-H_{\lambda}, Q, 0\right)$ is well defined for $\lambda \in I$. Moreover, under such conditions the homotopy invariance of the degree permits concluding that $d\left(I-H_{\lambda}, Q, 0\right)$ is constant. Therefore,

$$
d\left(I-H_{\lambda}, Q, 0\right)=d\left(I-H_{\lambda^{*}+1}, Q, 0\right)=0,
$$

the last identity coming from the fact that no solutions to (1.1) exist in $Q$ for $\lambda>\lambda^{*}$. Finally, the excision property implies

$$
d\left(I-H_{\bar{\lambda}_{1}}, B_{\varepsilon}, 0\right)=d\left(I-H_{\bar{\lambda}_{1}}, Q, 0\right)=0 .
$$

Since this contradicts 4.5 problem 1.1) must admit a second positive solution $v_{\bar{\lambda}_{1}}$ satisfying $v_{\bar{\lambda}_{1}} \geq u_{\bar{\lambda}_{1}}$.

The assertion in iv) concerning the minimal solution at $\lambda=\lambda^{*}$ is a consequence of the $L^{\infty}$ estimate in Theorem 6 . The family $\left\{u_{\lambda}\right\}$ is uniformly bounded in $C^{1, \beta}(\bar{\Omega})$ for all $0<\beta<1$. Thus, the limit

$$
u_{\lambda^{*}}:=\lim _{\lambda \rightarrow \lambda^{*}} u_{\lambda}=\sup _{0<\lambda<\lambda^{*}} u_{\lambda}
$$

holds in $C^{1, \beta}(\bar{\Omega})$ and defines a weak positive solution to $(1.1)$ for $\lambda=\lambda^{*}$. Schauder theory and the properties of $N_{f}$ as a Nemytskii operator taking values in $C^{\theta}(\bar{\Omega})$ for some $0<\theta<1$ (Lemma 3) permits upgrading the limit up to (at least) $C^{2}(\bar{\Omega})$. It is clear that $u_{\lambda^{*}}$ is the minimal solution to 1.1 at $\lambda=\lambda^{*}$.

Remark 3 . It will be shown in the next section that $u_{\lambda}$ is asymptotically stable for all $\lambda \in\left(0, \lambda^{*}\right)$ with the possible exception of $\lambda=\lambda_{n}$, with $\lambda_{n}$ a decreasing sequence satisfying $\lambda_{n} \rightarrow 0$. Therefore,

$$
d\left(I-H_{\lambda}, B\left(u_{\lambda}, \varepsilon\right), 0\right)=1,
$$

for $\varepsilon>0$ small and each $\lambda \neq \lambda_{n}$. This fact is coherent with 4.5. 


\section{Further PRoperties of The BRANCH OF Minimal SOLUTiONS $u_{\lambda}$}

The main purpose of this section is to prove Theorem 2. Our first result depicts the structure of the set of solutions to 1.1$)$ in the space $X \times(0, \infty)$ near a solution $(\bar{u}, \bar{\lambda})$, when $\bar{u}$ is positive and linearly stable, but in the critical case when the associated eigenvalue $\sigma_{1}=0$. As customary we set $\mathcal{P}=\{u \in X: u(x) \geq 0 x \in \Omega\}$ the positive cone in $X$ and $\stackrel{\circ}{\mathcal{P}}$ its interior relative to the $X$ topology. It is well-known that

$$
\stackrel{\circ}{\mathcal{P}}=\left\{u \in X: u(x)>0, x \in \Omega \text { and } \frac{\partial u}{\partial \nu}(x)<0, x \in \partial \Omega\right\} .
$$

The first eigenvalue to problem (2.7) will be designated, if necessary, by $\sigma_{1}(u)$ to stress its dependence on the function $u$.

Lemma 12. Let $(\bar{u}, \bar{\lambda})$ be a positive solution to 1.1 with $\sigma_{1}(\bar{u})=0$. Then there exists positive numbers $\varepsilon, \eta$ and $C^{\infty}$ mappings,

$$
\begin{array}{rlll}
\lambda:(-\varepsilon, \varepsilon) & \longrightarrow \mathbb{R} & u:(-\varepsilon, \varepsilon) & \longrightarrow \stackrel{\circ}{\mathcal{P}} \\
s & \longmapsto \lambda(s) & s & \longmapsto u_{s}=u(\cdot, s),
\end{array}
$$

verifying $u(\cdot, 0)=\bar{u}, \lambda(0)=\bar{\lambda}$ and such that,

i) $\left(u_{s}, \lambda(s)\right)$ solves (1.1) for every $-\varepsilon<s<\varepsilon$,

ii) if $(u, \lambda)$ solves (1.1) with $\|u-\bar{u}\|_{X}<\eta,|\lambda-\bar{\lambda}|<\eta$, then a unique $s \in(-\varepsilon, \varepsilon)$ exists such that $u=u_{s}$ and $\lambda=\lambda(s)$.

Moreover, $\lambda^{\prime}(0)=0, \lambda^{\prime \prime}(0)<0$ while $\sigma_{1}\left(u_{s}\right)>0$ (respectively, $\sigma_{1}\left(u_{s}\right)<0$ ) for $-\varepsilon<s<0(0<s<\varepsilon)$.

Remark 4. A solution $(\bar{u}, \bar{\lambda})$ exhibiting the properties in Lemma 12 is referred to as a "turning point" in the bifurcation diagram of solutions to (1.1) (see [25], [26]).

Proof of Lemma 12. According to the notation in Section 4 define,

$$
F(u, \lambda)=u-H(u, \lambda) .
$$

We now observe that the Nemytskii operator $N_{f}$ when considered as acting as $N_{f}: \stackrel{\circ}{\mathcal{P}} \rightarrow C(\bar{\Omega}), \stackrel{\circ}{\mathcal{P}}$ endowed with the topology of $X$, defines a class $C^{\infty}$ mapping such that,

$$
D_{u}^{k} N_{f}(u)\left(\hat{v}_{1}, \ldots, \hat{v}_{k}\right)=q(q-1) \cdots(q-k+1) u^{q-k} \hat{v}_{1} \ldots \hat{v}_{k},
$$

$\hat{v}_{1}, \ldots, \hat{v}_{k} \in X$, where $D_{u}^{k} N_{f}(u)$ stands for the $k$-th order Frechet differential of $N_{f}$ at $u$, acting as a $k$-linear operator. To show this assertion notice that a fixed function $u \in \stackrel{\circ}{\mathcal{P}}$ satisfies the estimates 2.6 while

$$
\left|\frac{v(x)}{d(x)}\right| \leq C\|v\|_{X} \quad x \in \Omega,
$$

for all $v \in X$ and a constant $C$ not depending on $v$ (recall the definition of the function $d(x)$ introduced in Section 2). Therefore $F: \stackrel{\circ}{\mathcal{P}} \times \mathbb{R} \rightarrow X$ defines a $C^{\infty}$ mapping.

As a consequence of Lemma 5 we know that

$$
N\left(D_{u} F(\bar{u}, \bar{\lambda})\right)=\operatorname{span}\{\phi\},
$$


where $\phi \in X$ is a positive eigenfunction associated to $\sigma_{1}(\bar{u})$, normalized in some way. Moreover, suppose that $\hat{w}=D_{u} F(\bar{u}, \bar{\lambda}) \hat{v}$ with $\hat{v}, \hat{w} \in X$. Then,

$$
\hat{v}-\hat{w}=\bar{\lambda} G\left(q \bar{u}^{q-1} \hat{v}\right),
$$

which means that

$$
\int_{\Omega} \nabla \hat{v} \nabla \varphi-\int_{\Omega} \nabla \hat{w} \nabla \varphi=\bar{\lambda} \int_{\Omega} q \bar{u}^{q-1} \hat{v} \varphi
$$

for each $\varphi \in H_{0}^{1}(\Omega)$. Taking into account that $\sigma_{1}(\bar{u})=0$, i.e.,

$$
\int_{\Omega} \nabla \phi \nabla \varphi=\bar{\lambda} \int_{\Omega} q \bar{u}^{q-1} \phi \varphi
$$

for each $\varphi \in H_{0}^{1}(\Omega)$, we conclude that,

$$
\int_{\Omega} \nabla \hat{w} \nabla \phi=0
$$

Thus, relation (5.6) characterizes the fact that $\hat{w}$ lies in the range of the operator $D_{u} F(\bar{u}, \lambda)$. Since $\hat{w}=D_{\lambda} F(\bar{u}, \bar{\lambda})=-G\left(\bar{u}^{q}\right)$ does not satisfy such relation, the existence of functions $u_{s}=u(\cdot, s)$ and $\lambda(s)$ verifying assertions i), ii) together with $\lambda^{\prime}(0)=0$ follows from [26] (see Chapter 1). Moreover,

$$
u(\cdot, s)=\bar{u}+\phi s+o(s),
$$

as $s \rightarrow 0$ in $X$. Accordingly, $u(\cdot, s)$ can be chosen increasing in $(-\varepsilon, \varepsilon)$. As for the assertion concerning the sign of $\lambda^{\prime \prime}(0)$ we follow ideas from [25]. By differentiating the equality,

$$
F\left(u_{s}, \lambda(s)\right)=0,
$$

twice with respect to $s$ and then setting $s=0$ we obtain:

$$
D_{u}^{2} F(\bar{u}, \bar{\lambda})(\phi, \phi)+D_{u} F(\bar{u}, \bar{\lambda})\left(u^{\prime \prime}(0)\right)+D_{\lambda} F(\bar{u}, \bar{\lambda}) \lambda^{\prime \prime}(0)=0,
$$

with $u^{\prime \prime}(0)$ the second derivative of $u_{s}$ at $s=0$. Using (5.6) we find,

$$
\lambda^{\prime \prime}(0)=-\frac{\int_{\Omega} \nabla\left\{D_{u}^{2} F(\bar{u}, \bar{\lambda})(\phi, \phi)\right\} \nabla \phi}{\int_{\Omega} \nabla\left\{D_{\lambda} F(\bar{u}, \bar{\lambda})\right\} \nabla \phi} .
$$

Now observe that $\int_{\Omega} \nabla\left\{D_{\lambda} F(\bar{u}, \bar{\lambda})\right\} \nabla \phi=-\int_{\Omega} \bar{u}^{q} \phi$ while

$$
\int_{\Omega} \nabla\left\{D_{u}^{2} F(\bar{u}, \bar{\lambda})(\phi, \phi)\right\} \nabla \phi=-\bar{\lambda} \int_{\Omega} q(q-1) \bar{u}^{q-2} \phi^{3} .
$$

Accordingly,

$$
\lambda^{\prime \prime}(0)=-\frac{\bar{\lambda} \int_{\Omega} q(q-1) \bar{u}^{q-2} \phi^{3}}{\int_{\Omega} \bar{u}^{q} \phi} .
$$

On the other hand, Piccone's identity ([25]) states that

$$
\int_{\Omega} h\left(\frac{\phi}{\bar{u}}\right)(\phi \Delta \bar{u}-\bar{u} \Delta \phi)=\int_{\Omega} \bar{u}^{2} h^{\prime}\left(\frac{\phi}{\bar{u}}\right)\left|\nabla\left(\frac{\phi}{\bar{u}}\right)\right|^{2},
$$

with $h$ an arbitrary $C^{1}$ function. Choosing $h(t)=t^{2}$ we get that

$$
\bar{\lambda} \int_{\Omega}(q-1) \bar{u}^{q-2} \phi^{3}>0 .
$$


That $\lambda^{\prime \prime}(0)<0$ then follows from the equality $q(q-1)=(q-1)^{2}+(q-1)$. In particular, the function $\lambda(s)$ can be chosen increasing in $(-\varepsilon, 0)$ and decreasing in $(0, \varepsilon)$.

Concerning the sign of $\sigma_{1}(s)$, it is classical (cf. [12]) that both $\sigma_{1}(\bar{u})$ and $\phi$ can be perturbed to obtain smooth mappings $\sigma_{1}(s):=\sigma_{1}\left(u_{s}\right), \phi(s)$ such that

$$
D_{u}\left(u_{s}, \lambda(s)\right)(\phi(s))=\sigma_{1}(s) \phi(s),
$$

for $|s|<\varepsilon, \varepsilon>0$ small. Taking derivatives with respect to $s$, setting $s=0$ and using that $\lambda^{\prime}(0)=0, \sigma_{1}(0)=\sigma_{1}(\bar{u})=0$ we obtain

$$
D_{u}^{2} F(\bar{u}, \bar{\lambda})(\phi, \phi)+D_{u} F(\bar{u}, \bar{\lambda})\left(\phi^{\prime}(0)\right)=\sigma_{1}^{\prime}(0) \phi .
$$

Taking gradients, performing a scalar product with $\nabla \phi$ and integrating in $\Omega$ gives

$$
\sigma_{1}^{\prime}(0)=\frac{\int_{\Omega} q \bar{u}^{q} \phi}{\bar{\lambda} \int_{\Omega} q \bar{u}^{q-1} \phi^{2}} \lambda^{\prime \prime}(0)<0 .
$$

Thus the assertions concerning the sign of $\sigma_{1}(s)$ hold, provided $\varepsilon>0$ is suitably reduced.

Lemma 13. Suppose $(\bar{u}, \bar{\lambda})$ is a positive solution to (1.1) with $\sigma_{1}(\bar{u}) \geq 0$. Then, there exists an increasing family $\left\{\left(\hat{u}_{\lambda}, \lambda\right)\right\}, 0<\lambda \leq \bar{\lambda}$ of positive solutions to (1.1) so that $\lambda \mapsto \tilde{u}_{\lambda}$ is smooth in $(0, \bar{\lambda}), \sigma_{1}\left(\hat{u}_{\lambda}\right)>0$ for each $\lambda \in(0, \bar{\lambda})$ and $\hat{u}_{\lambda} \rightarrow 0$ as $\lambda \rightarrow 0+$.

Proof. Let us first assume that $\sigma_{1}(\bar{u})>0$. Then, $D_{u} F(\bar{u}, \bar{\lambda})$ admits an order preserving inverse. The implicit function theorem then implies that (1.1) is uniquely solvable near $(\bar{u}, \bar{\lambda})$ in $\stackrel{\circ}{\mathcal{P}} \times(0, \infty)$ by a smooth family $\left\{\left(\hat{u}_{\lambda}, \lambda\right)\right\}$, with $\bar{\lambda}-\delta<\lambda<\bar{\lambda}+\delta$, being $\sigma_{1}\left(\hat{u}_{\lambda}\right)>0$. Therefore, $\hat{u}_{\lambda}$ is increasing in $\lambda$.

Assume that $\bar{\lambda}-\delta>0$. Then, the limit

$$
\lim _{\lambda \rightarrow(\bar{\lambda}-\delta)+} \hat{u}_{\lambda}:=\hat{u}
$$

is finite and satisfies:

$$
\hat{u} \geq \tilde{u}_{\bar{\lambda}-\delta}>0,
$$

where $\tilde{u}_{\lambda}$ is the solution to (2.2). Thus $\hat{u}$ defines a positive solution to (1.1) at $\lambda=\bar{\lambda}-\delta$. In addition $\sigma_{1}(\hat{u}) \geq 0$. The case $\sigma_{1}(\hat{u})=0$ can be ruled out since Lemma 12 would imply that $(\hat{u}, \bar{\lambda}-\delta)$ is isolated from the right of $\bar{\lambda}$ that is not true. Therefore $\sigma_{1}(\hat{u})>0$. A new iteration of the process permits a further continuation of $\hat{u}_{\lambda}$ to the left of $\bar{\lambda}-\delta$. In this way, $\lambda \mapsto \hat{u}_{\lambda}$ can be defined as an increasing smooth mapping in the whole interval $(0, \bar{\lambda}+\delta)$ which fulfils condition $\sigma_{1}\left(\hat{u}_{\lambda}\right)>0$. The convergence $\hat{u}_{\lambda} \rightarrow 0$ as $\lambda \rightarrow 0$ is a consequence of ii) in Theorem 1 .

As for the case $\sigma_{1}(\bar{u})=0$, Lemma 12 provides the existence of a family $\hat{u}_{\lambda}$ of positive solutions which is smooth in $\bar{\lambda}-\varepsilon<\lambda<\bar{\lambda}$, continuous in $\bar{\lambda}-\varepsilon<\lambda \leq \bar{\lambda}$, increasing in $\lambda$, satisfies $\sigma_{1}\left(\hat{u}_{\lambda}\right)>0$ for $\lambda<\bar{\lambda}$ and $\hat{u}_{\lambda}=\bar{u}$ at $\lambda=\bar{\lambda}$. The previous argument can now be employed to extend $\hat{u}_{\lambda}$ up to $\lambda=0$

Lemma 14. The branch of minimal solutions $u_{\lambda}$ is discontinuous at $\lambda=$ $\bar{\lambda} \in\left(0, \lambda^{*}\right)$ if and only if $\sigma_{1}\left(u_{\bar{\lambda}}\right)=0$. 
Proof. If $\sigma_{1}\left(u_{\bar{\lambda}}\right)=0$ then $\left(u_{\bar{\lambda}}, \bar{\lambda}\right)$ defines a turning point and therefore (Lemma 12 it is isolated from the right of $\bar{\lambda}$, i. e. there exists $U$, a neighborhood of $u_{\bar{\lambda}}$ in $\stackrel{\circ}{\mathcal{P}}$ and $\varepsilon>0$ so that there exist no solutions $(u, \lambda) \in$ $U \times(\bar{\lambda}, \bar{\lambda}+\varepsilon)$ to (1.1). Thus, $\lim _{\lambda \rightarrow \bar{\lambda}+} u_{\lambda}>u_{\bar{\lambda}}$ and $u_{\lambda}$ is discontinuous at $\lambda=\bar{\lambda}$.

Now assume that $u_{\lambda}$ is discontinuous at $\lambda=\bar{\lambda}$. Since $\sigma_{1}\left(u_{\bar{\lambda}}\right) \geq 0$ we rule out the case $\sigma_{1}\left(u_{\bar{\lambda}}\right)>0$. In fact, if such fact holds then Lemma 13 furnishes a smooth family $\hat{u}_{\lambda}$ of positive solutions such that $\hat{u}_{\lambda}=u_{\bar{\lambda}}$ for $\lambda=\bar{\lambda}$. Since $u_{\lambda}$ is continuos at $\bar{\lambda}$ from the left we obtain that $\hat{u}_{\lambda}=u_{\lambda}$ for $\bar{\lambda}-\varepsilon<\lambda \leq \bar{\lambda}$. On the other hand, discontinuity of $u_{\lambda}$ from the left of $\bar{\lambda}$ implies that $u_{\bar{\lambda}}^{+}:=\lim _{\lambda \rightarrow \bar{\lambda}+} u_{\lambda}>u_{\bar{\lambda}}$, in the sense that even $u_{\bar{\lambda}}^{+}-u_{\bar{\lambda}} \in \stackrel{\circ}{\mathcal{P}}$. This entails that $\hat{u}_{\lambda}<u_{\lambda}$ for $\bar{\lambda}<\lambda<\bar{\lambda}+\varepsilon$ and a certain small $\varepsilon>0$. This is not possible since $u_{\lambda}$ is the minimal solution.

Lemma 15. For every $\lambda_{0} \in\left(0, \lambda^{*}\right)$ the branch of minimal solutions $u_{\lambda}$ is discontinuous at most at a finite number of values $\lambda_{0} \leq \lambda<\lambda^{*}$.

Proof. Assume, with no loss of generality, the existence of a decreasing sequence $\lambda_{n}$ such that $\lambda_{n} \rightarrow \lambda_{0}$ for some $\lambda_{0}>0$, being $\lambda=\lambda_{n}$ a discontinuity point of $u_{\lambda}$. Since every $\left(\lambda_{n}, u_{\lambda_{n}}\right)$ is a turning point, Lemmas 13 and 14 assert the existence of a smooth increasing family $\hat{u}_{\lambda}^{(n)}$ of positive solutions connecting $\left(\lambda_{n}, u_{\lambda_{n}}\right)$ with $(0,0)$ in $\stackrel{\circ}{\mathcal{P}} \times\left[0, \lambda^{*}\right)$. Let us set now $u_{n}=\hat{u}_{\lambda_{0}}^{(n)}$. Then, $u_{n}$ defines a family of positive solutions to (1.1), $\lambda=\lambda_{0}$, satisfying

$$
\tilde{u}_{\lambda_{0}} \leq u_{n} \leq u_{\lambda_{n}} \leq u_{\lambda_{1}}
$$

where $\tilde{u}_{\lambda}$ is the family of positive solutions to 2.2 . Since $\left\|u_{n}\right\|_{L^{\infty}(\Omega)} \leq M$, $u_{n}$ converges to a positive solution $u_{0}$ to (1.1) with $\lambda=\lambda_{0}$. On the other hand, $\sigma_{1}\left(u_{n}\right)>0$. Thus $\sigma_{1}\left(u_{0}\right) \geq 0$. However, if either $\sigma_{1}\left(u_{0}\right)>0$ or $\sigma_{1}\left(u_{0}\right)=0, u_{0}$ becomes an isolated positive solution to (1.1). This is not compatible with the definition of $u_{0}$. Therefore, $u_{\lambda}$ exhibits at most a finite number of discontinuities for $\lambda \geq \lambda_{0}>0$.

Proof of Theorem 2. As a first remark, Lemma 15 implies that $u_{\lambda}$ is at most discontinuous in a decreasing sequence $\lambda_{n}$ in the interval $\left(0, \lambda^{*}\right)$. Fix now $n$. Lemma 13 ensures the existence of a smooth increasing family of positive solutions $\hat{u}_{\lambda}$, connecting $u=0$ at $\lambda=0$ with $u_{\lambda_{n}}$ at $\lambda=\lambda_{n}$ and fulfilling $\sigma_{1}\left(\hat{u}_{\lambda}\right)>0$. Since $u_{\lambda}$ is continuos from the left at $\lambda=\lambda_{n}$, it follows from the uniqueness assertion in Lemma 12 that $\hat{u}_{\lambda}=u_{\lambda}$ for $\lambda_{n+1} \leq \lambda_{n}-\varepsilon<\lambda \leq \lambda_{n}$ for a small $\varepsilon>0$.

Set $\hat{\lambda}=\inf \left\{\lambda: \lambda_{n+1}<\lambda<\lambda_{n}, \hat{u}_{\lambda}=u_{\lambda}\right\}$. The uniqueness assertion in the implicit function theorem then implies that $\sigma_{1}\left(u_{\hat{\lambda}}\right)=0$. But then $u_{\lambda}$ is discontinuous at $\lambda=\hat{\lambda}$ (Lemma 14) and thus $\hat{\lambda}=\lambda_{n+1}$. This shows ii) and the proof is finished.

\section{ACKNOWLEDGEMENTS}

Supported by Spanish Ministerio de Ciencia e Innovación and Ministerio de Economía y Competitividad under grant reference MTM2011-27998. 


\section{REFERENCES}

[1] H. Amann, Fixed point equations and nonlinear eigenvalue problems in ordered Banach spaces, SIAM Rev. 18 (1976), no. 4, 620-709.

[2] H. Amann, J. LóPEz-Gómez, A priori bounds and multiple solutions for superlinear indefinite elliptic problems, J. Diff. Eqns. 146 (1998), 336-374.

[3] A. Ambrosetti, H. Brezis, G. Cerami, Combined effects of concave and convex nonlinearities in some elliptic problems, J. Funct. Anal. 122 (1994), 519-543.

[4] A. Ambrosetti, J. García-Azorero, I. Peral, Multiplicity results for some nonlinear elliptic equations, J. Funct. Anal. 137 (1996), 219-242.

[5] L. Boccardo, M. Escobedo, I. Peral, A Dirichlet problem involving critical exponents, Nonlinear Anal. 24 (1995), no. 11, 1639-1648.

[6] H. Brezis, Functional Analysis, Sobolev Spaces and Partial Differential Equations, Springer, New York, 2011.

[7] H. Brezis, L. Nirenberg, $H^{1}$ versus $C^{1}$ local minimizers, C. R. Acad. Sci. Paris Sér. I Math. 317 (1993), no. 5, 465-472.

[8] H. BREzIs, T. KATO, Remarks on the Schrödinger operator with singular complex potentials, J. Math. Pures Appl. (9) 58 (1979), no. 2, 137-151.

[9] H. Brezis, L. Oswald, Remarks on sublinear elliptic equations, Nonlinear Anal. 10 (1986), no. 1, 55-64.

[10] F. Charro, E. Colorado, I. Peral, Multiplicity of solutions to uniformly elliptic Fully Nonlinear equations with concave-convex right hand side, J. Diff. Eqns. 246 (2009), 4221-4248.

[11] C. Cortázar, M. Elgueta, P. Felmer, On a semilinear elliptic problem in $\mathbb{R}^{N}$ with a non-Lipschitzian nonlinearity, Adv. Differential Equations 1 (1996), no. 2, 199-218.

[12] M. G. Crandall, P. H. Rabinowitz, Bifurcation, perturbation of simple eigenvalues and linearized stability, Arch. Rational Mech. Anal. 52 (1973), 161-180.

[13] M. Delgado, J. López-Gómez, A. SuÁrez, Combining linear and non-linear diffusion, Adv. Nonl. Stud. 4 (2004), 273-287.

[14] P. C. Fife, Mathematical aspects of reacting and diffusing systems. Lecture Notes in Biomathematics no. 28, Springer, Berlin, 1979.

[15] J.P. García Azorero, J.J. Manfredi, I. Peral Alonso, Sobolev versus Hölder local minimizers and global multiplicity for some quasilinear elliptic equations, Comm. Contemp. Maths. 2 (2000), 385-404.

[16] J. García-Azorero, I. Peral Alonso, Multiplicity of solutions for elliptic problems with critical exponent or with a nonsymmetric term, Trans. Amer. Math. Soc. 323 (1991), 877-895.

[17] J. García-Azorero, I. Peral Alonso, Some results about the existence of a second positive solution in a quasilinear critical problem, Indiana Univ. Math. J. 43 (1994), no. 3, 941-957.

[18] J. García-Azorero, I. Peral, J. D. Rossi, A convex-concave problem with a nonlinear boundary condition, J. Diff. Eqns. 198 (1) (2004), 91-128.

[19] J. García-Melián, J.D. Rossi, J. Sabina de Lis, Large solutions for the Laplacian with a power nonlinearity given by a variable exponent, Ann. Inst. H. Poincaré Anal. Non Linéaire 26 (2009), 889-902.

[20] J. García-Melián, J.D. Rossi, J. Sabina de Lis, Existence, asymptotic behavior and uniqueness for large solutions to $\Delta u=e^{q(x) u}$, Adv. Nonlinear Stud. 9 (2) (2009), 395-424.

[21] J. García-Melián, J. D. Rossi, J. Sabina de Lis, A convex-concave elliptic problem with a parameter on the boundary condition, Discrete Contin. Dyn. Syst. 32 (2012), no. 4, 1095-1124.

[22] B. Gidas, J. Spruck, Global and local behavior of positive solutions of nonlinear elliptic equations. Comm. Pure Appl. Math. 34 (1981), 525-598.

[23] B. Gidas, J. Spruck, A priori bounds for positive solutions of nonlinear elliptic equations, Comm. Partial Differential Equations 6 (1981), no. 8, 883-901. 
[24] D. Gilbarg, N.S. Trudinger, Elliptic partial differential equations of second order. Springer-Verlag, 1983.

[25] R. Gómez-Reñasco, J. López-Gómez, The effect of varying coefficients on the dynamics of a class of superlinear indefinite reaction-diffusion equations, J. Diff. Eqns. 167 (2000), no. 1, 36-72.

[26] H. KiELhÖFER, Bifurcation theory. An introduction with applications to PDEs. Springer-Verlag, New York, 2004.

[27] A. V. Lair, A. Mohammed, Entire large solutions to elliptic equations of power non-linearities with variable exponents. Adv. Nonl. Stud. 13 (2013), no. 3, 699-719.

[28] P. Lindqvist, On the equation $\operatorname{div}\left(|\nabla u|^{p-2} \nabla u\right)+\lambda|u|^{p-2} u=0$, Proc. Amer. Math. Soc. 109 (1990), 157-164.

[29] P. L. Lions, On the existence of positive solutions of semilinear elliptic equations. SIAM Rev. 24 (1982), 441-467.

[30] J. LóPEz-Gómez, Varying stoichometric exponents I: Classical steady states and metasolutions, Adv. Nonl. Stud. 3 (2003), 327-354.

[31] J. López-Gómez, A. SuÁrez, Combining fast, linear and slow diffusion, Topol. Methods Nonl. Anal. 23 (2004), 275-300.

[32] F. Mignot, J. P. Puel, Sur une classe de problèmes nonlinéaires avec nonlinéarité positive, croissante, convexe. Comm. Partial Differential Equations 5 (1980), 791836.

[33] W. M. NI, The mathematics of diffusion, CBMS-NSF Regional Conference Series in Applied Mathematics, 82. Society for Industrial and Applied Mathematics (SIAM), Philadelphia, 2011.

[34] C. V. PAO, Nonlinear parabolic and elliptic equations, Plenum Press, New York, 1992.

[35] P. Pucci, Q. Zhang, Existence of entire solutions for a class of variable exponent elliptic equations, J. Diff. Eqns. 257 (2014), 1529-1566.

[36] D. RuIz, A priori estimates and existence of positive solutions for strongly nonlinear problems, J. Diff. Eqns. 199 (2004), 96-114.

[37] J. SABInA DE LIS, S. SEgURA DE LEÓN, Multiplicity of solutions to a nonlinear boundary value problem of concave-convex type, to appear in Adv. Nonlinear Studies.

[38] J. SERRIN, Local behavior of solutions of quasilinear equations, Acta Math. 111 (1964), 247-302.

[39] J. Serrin, H. Zou, Cauchy-Liouville and universal boundedness theorems for quasilinear elliptic equations and inequalities, Acta Math. 189 (2002), 79-142.

[40] J. Smoller, Shock waves and reaction-diffusion equations, Springer-Verlag, New York, 1994.

[41] M. Struwe, Variational methods. Applications to nonlinear partial differential equations and Hamiltonian systems, Springer-Verlag, Berlin, 2008.

J. García-Melián and J. C. Sabina de Lis

Departamento de AnÁlisis Matemático, Universidad de la laguna. C/. Astrofísico Francisco Sánchez s/n, 38271 - La Laguna, SPAin. and

Instituto Universitario de Estudios Avanzados (IUdEA) en Física Atómica, Molecular y Fotónica, Universidad de La Laguna

C/. Astrofísico Francisco SÁnchez s/n, 38203 - La Laguna, SPAin.

E-mail address: jjgarmel@ull.es, josabina@ull.es

J. D. Rossi

Departamento de Matemática, FCEyn UBA,

Ciudad Universitaria, Pab 1 (1428),

Buenos Aires, ARGENTINA.

E-mail address: jrossi@dm.uba.ar 